\section{En acción: mejorando el acceso a la atención óptima para todos los pacientes con inmunodeficiencias primarias Semana mundial de las Inmunodeficiencias Primarias (WPIW) 22-29 de abril, 2016}

\author{
In action: Improving \\ access to optimal care for \\ all patients with primary \\ immunodeficiencies \\ Global Week of primary \\ immunodeficiencies ( WPIW ) \\ April 22-29, 2016
}

Las inmunodeficiencias primarias constituyen un grupo de más de 300 defectos innatos del sistema inmunitario, tanto en sus componentes hematopoyéticos como en los no-hematopoyéticos. Dichos defectos pueden presentarse con un amplio espectro de manifestaciones clínicas, ya sea con infecciones (comunes recurrentes, comunes graves, o raras y graves), autoinflamación, autoinmunidad, malignidad y/o alergia. Anteriormente clasificadas como "enfermedades raras", las inmunodeficiencias primarias no son tan raras como se pensaba: hoy en día se estima que afectan a más de seis millones de personas alrededor del mundo, sin distinciones geográficas, de género o edad.
Debido a que las inmunodeficiencias primarias pueden presentarse bajo la forma de infecciones "comunes", la falta de conocimiento y familiaridad genera que entre 70 y $90 \%$ de personas afectadas se quede sin diagnóstico, toda vez que sus profesionales de la salud tratan dichas infecciones como cualquiera otra "infección común". En respuesta a la necesidad urgente de despertar conciencia acerca de esta condición, organizaciones preocupadas como la Sociedad Africana para las Inmunodeficiencias (ASID), Sociedad Europea para Inmunodeficiencias (ESID), la Sociedad Latinoamericana para Inmunodeficiencias (LASID), la Sociedad de Inmunología Clínica (CIS) y funda- 
ciones dedicadas a inmunodeficiencias primarias tales como: la Fundación de Deficiencia Inmune (IDF) y la Fundación Jeffrey Modell (JMF), así como organizaciones de pacientes como la Organización de Pacientes para Inmunodeficiencias Primarias (IPOPI), han implementado y apoyado, a lo largo de más de dos décadas, campañas y actividades a todo lo largo del mundo, dirigidas a médicos y al público en general, para incrementar el diagnóstico temprano, tratamiento apropiado y el manejo continuado de las inmunodeficiencias primarias, con miras a reducir la morbilidad y mortalidad asociadas a estos defectos.

Desde el 2011 se inició una campaña global anual -la Semana Mundial de las Inmunodeficiencias Primarias- para incrementar el conocimiento y familiaridad bajo el Ilamado a la acción: "Evalúa. Diagnostica. Trata.", y se celebra cada abril con el apoyo de una red global de partes interesadas en inmunodeficiencias primarias (www.worldpiweek.org), para acercar todos los grupos activos en el campo alrededor de una plataforma común, y así fortalecer el impacto de sus actividades a nivel local.

Como resultado de dichas actividades, y gracias a un número creciente de tratamientos capaces de salvar vidas que han sido desarrollados a lo largo de los últimos 60 años, el número de pacientes con inmunodeficiencias primarias diagnosticados y tratados sigue creciendo cada año. Sin embargo, a pesar de estos progresos, resulta profundamente preocupante que el acceso al tratamiento varíe dramáticamente a través de los continentes, e incluso de manera significativa a través de países en una misma región. Por ejemplo, el pronto acceso a un trasplante de células madre y a terapia génica para varios tipos de inmunodeficiencias primarias es la regla en la mayoría de países de Norteamérica y Europa, mientras que está disponible en apenas un número muy pequeño de centros en Latinoamérica y África. ¿Es esto aceptable? De manera similar, mientras que en la mayoría de países europeos los sistemas de salud nacionales proporcionan o reembolsan el tratamiento con inmunoglobulina G (IgG) intravenosa o subcutánea, estos tratamientos no siempre son cubiertos en países más pobres, a pesar de que existe evidencia abundante de que el diagnóstico temprano y el manejo apropiado de pacientes con inmunodeficiencias primarias salvan vidas, mejoran la vida y ahorran costos, además de prevenir morbilidad innecesaria.

En vista de tales discrepancias, este año las partes interesadas, unidas bajo el estandarte de la Semana Mundial de las Inmunodeficiencias Primarias, levantan sus voces una vez más, para convocar a tomadores de decisiones, autoridades de salud, financiadores de sistemas de atención y profesionales de la salud, a que se implementen los mecanismos relevantes que garanticen que todos los pacientes puedan recibir tratamientos apropiados, seguros y eficientes; así como un cuidado óptimo a todo lo ancho del mundo, tanto por su propio beneficio, como para un beneficio a largo plazo del sistema de salud.

Los pacientes con inmunodeficiencias primarias requieren acceso expedito a antibióticos antifúngicos y antibacterianos profilácticos, y un acceso continuo al tratamiento de reemplazo con inmunoglobulina. Además, algunos pacientes requieren trasplante de células madre hematopoyéticas o incluso terapia génica, así como medicina de emergencia de acuerdo a sus necesidades y siguiendo las recomendaciones de sus especialistas.

Como tratamiento incluido en la Lista de la Organización Mundial de la Salud de Medicamentos Esenciales para niños y adultos, y al ser un tratamiento efectivo que salva vidas en la mayoría de pacientes con inmunodeficiencias primarias, la IgG debería facilitarse a estos pa- 
cientes en todo el mundo. Más específicamente, dado que no hay un solo producto o método de administración de $\operatorname{lgG}$ que sea apropiado para todos los pacientes, todos los países y centros deberían tener acceso a una gama amplia de productos de IgG para poder proporcionar un manejo óptimo a cada paciente. Deberían implementarse asimismo mecanismos de fondeo y financiamiento que garanticen la disponibilidad y la dosificación óptima de la lgG, y que de esa manera mejoren la calidad de vida de los pacientes, evitando un mayor daño orgánico y reduciendo los costos de tratamiento.

Más aún, todos los pacientes que lo necesiten deberían recibir trasplante de células madre hematopoyéticas o terapia génica sin importar dónde vivan. Se ha demostrado que el trasplante temprano cura varias de las formas más graves de inmunodeficiencia primaria. El tamizaje neonatal sistemático para inmunodeficiencia combinada grave y linfopenia severa de células T, usando el método de cuantificación de cír-

Varios estudios han tratado de establecer una prevalencia de inmunodeficiencias primarias en varios países, y han generado resultados inconsistentes: se estimó una frecuencia de 0.38/100,000 habitantes en México 2007; 1.94/100,000 en Reino Unido 2011; 3.3/100,000 en Argentina 2007; 5.38/100,000 en Francia 2011 y 5.6/100,000 en Australia 2007. Basados en datos de registros, estos estimados parecen mucho menores que algunos otros basados en encuestas de poblaciones específicas recientemente recabados en los Estados Unidos de América, incluyendo la encuesta telefónica llevada a cabo por Boyle\&Buckley que reportó una prevalencia de 86.3/100,000 habitantes. ${ }^{1-3}$ culos de excisión de células T, está disponible en muy pocos países alrededor del mundo, pero ha mostrado ser capaz de reducir dramáticamente el costo de un trasplante de células madre hematopoyéticas.

Desafortunadamente, a causa de la falta de infraestructura $y / 0$ de personal calificado, el acceso a estos procedimientos es muy difícil en escenarios de menos recursos, y el tiempo de espera es con frecuencia tan largo, que muchos pacientes sufren complicaciones graves debidas a infecciones recurrentes. La falta de un donador compatible es también un problema común. Los registros de donadores de médula ósea deberían ser promovidos en cada país para incrementar las probabilidades de realizar un trasplante de células madre hematopoyéticas con la mejor compatibilidad y por lo tanto el mejor resultado.

Existen varias formas de inmunodeficiencias primarias letales y graves, en las cuales la terapia génica ha demostrado ser efectiva y segura, por lo que se está convirtiendo en el procedimiento estándar, especialmente en casos en los que no hay disponible un donador idéntico. Desgraciadamente, actualmente se realiza sólo en muy pocos centros altamente especializados, en países de ingresos elevados. Las autoridades legislativas y sanitarias tendrían que invertir en esta tecnología para garantizar su disponibilidad cuando no es factible realizar un trasplante de células madre hematopoyéticas.

Más allá de políticas nacionales, se deben implementar mecanismos de colaboración internacional entre centros de referencia establecidos y países que carecen de los requerimientos financieros o técnicos para proporcionar terapias de IgG, trasplante de células madre hematopoyéticas, terapia génica, u otros tratamientos salvadores. 
De manera adicional, las autoridades de salud en cada país deberían promover el acceso a diagnósticos tempranos y a una atención especializada de calidad, para asegurar un tratamiento apropiado para todos los pacientes. Los programas de concientización resultan en más pacientes con inmunodeficiencias primarias diagnosticados cada año, con lo cual la infraestructura existente está siendo saturada, y muchos pacientes deben viajar distancias largas para recibir atención médica adecuada. Deberían crearse además nuevos centros regionales especializados, que permitan un acceso geográfico equitativo a personal médico y de enfermería experto en estas enfermedades; más doctores especializados en este campo deberán ser entrenados para diagnosticar y dar atención médica adecuada a ese número creciente de pacientes. Al mismo tiempo, para los pacientes pediátricos que alcanzan la edad adulta, se deben establecer rutas coordinadas de transición hacia servicios de calidad para adultos que aseguren una atención planificada.

Debido a su papel en proporcionar información de utilidad para apoyar a los médicos en la toma de decisiones, y en permitir que las compañías farmacéuticas garanticen el abasto de productos médicos relevantes para cubrir la demanda, deben establecerse registros de pacientes que nos ayuden a evaluar la prevalencia y la incidencia de la inmunodeficiencia primaria. También se deben implementar registros internacionales de pacientes que proporcionen información a los centros de países en desarrollo que no tienen redes nacionales propias.

Por último, si bien no menos importante, necesitamos promover la formación de redes profesionales que utilicen los recursos de centros especializados certificados y registros de pacientes, que establezcan lineamientos de manejo y eleven los estándares de la atención, que desarrollen grupos organizados de comunicación en línea para discutir casos clínicos difíciles, y así apoyar a médicos que viven lejos de los centros especializados.

Con diagnóstico oportuno, atención adecuada y tratamientos óptimos, las inmunodeficiencias primarias son manejables y es posible evitar discapacidades permanentes, vacunaciones innecesarias y hospitalizaciones prolongadas a los pacientes. Por ello es vital que se tomen pasos decisivos en los niveles local, regional e internacional, para garantizar que los mecanismos arriba mencionados sean puestos en marcha, y permitir que todos los pacientes tengan acceso a una atención óptima a la que tienen derecho.

\section{Agradecimientos}

Saúl Oswaldo Lugo Reyes traductor de esta versión del original en inglés.

\section{LECTURAS RECOMENDADAS}

1. Bousfiha AA, Jeddane L, Ailal F, Benhsaien I, Mahlaoui N, Casanova JL, Abel L. Primary immunodeficiency diseases worldwide: more common than generally thought. J ClinImmunol. 2013 Jan;33(1):1-7.

2. Errante PR, Franco JL, Espinosa-Rosales FJ, Sorensen R, Condino-Neto A. Advances in primary immunodeficiency diseases in Latin America: epidemiology, research, and perspectives. Ann N Y Acad Sci. 2012;1250:62-72.

3. Boyle JM, Buckley RH. Population prevalence of diagnosed primary immunodeficiency diseases in the United States. J Clinlmmunol. 2007;27(5):497-502.

4. http://www.who.int/medicines/publications/essentialmedicines/EMLc_2015_FINAL_amended_AUG2015.pdf?ua=1 consulted on November 20, 2015.

5. http://www.who.int/selection_medicines/committees/expert/20/EML_2015_FINAL_amended_AUG2015.pdf?ua=1 consulted on November 20, 2015.

6. Clément MC, Mahlaoui N, Mignot C, Le Bihan C, Rabetrano H, Hoang L, Neven B, Moshous D, Cavazzana M, Blanche S, Fischer A, Audrain M, Durand-Zaleski I. Systematic neonatal screening for severe combined immunodeficiency and severe T-cell lymphopenia: Analysis of cost-effectiveness based on French real field data. J Allergy ClinImmunol2015;135:1589-93. 
7. Ghosh S, Thrasher AJ, Gaspar HB. Gene therapy for monogenic disorders of the bone marrow. $\mathrm{Br} J$ Haematol. 2015 Jun 5. doi: 10.1111/bjh.13520 [Epub ahead of print]

8. Principles of Care for Primary Immunodeficiencies http://journal.frontiersin.org/article/10.3389/ fimmu.2014.00627/full

Francisco J. Espinosa-Rosales Unidad de Inmunología y Alergia Instituto Nacional de Pediatría Ciudad de México, México.

\section{Antonio Condino-Neto}

Departamento de Inmunología Instituto de Ciencias Biomédicas
José L. Franco

Grupo de Inmunodeficiencias Primarias Escuela de Medicina, Universidad de Antioquía Medellín, Colombia.

Ricardo U. Sorensen

Departamento de Pediatría Louisiana State University Health Science

Center

New Orleans, LA.

Profesorado Honorario de la Facultad de Medicina

Universidad de La Frontera,

Temuco, Chile.

Universidad de Sao Paulo, Sao Paulo, SP, Brasil.

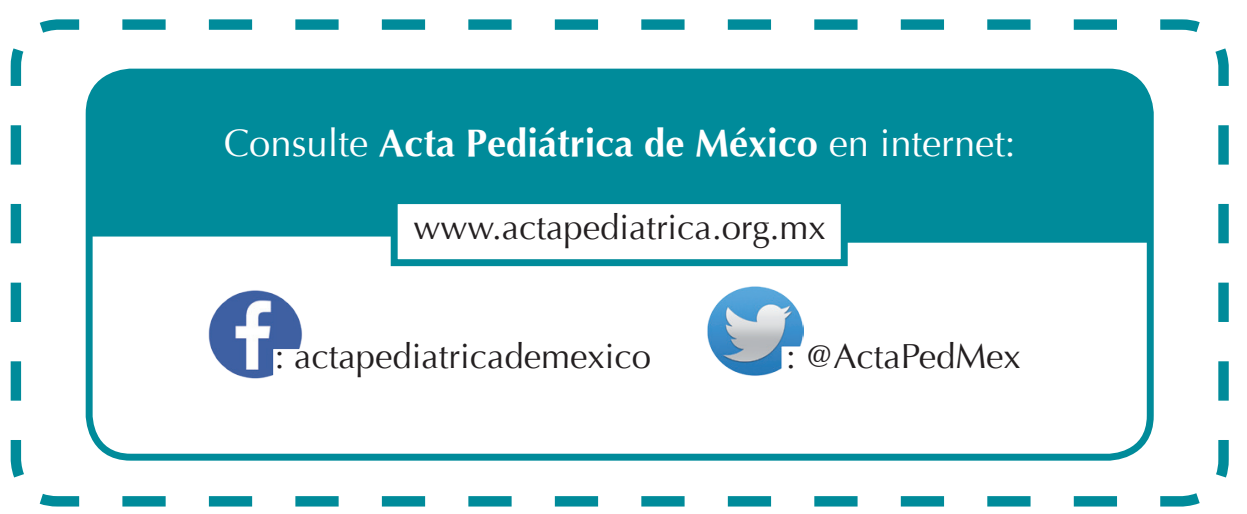

